\title{
Functional connectivity and grey matter volume of the striatum in schizophrenia
}

Kathrin Koch, Oana Georgiana Rus, Tim Jonas Reeß, Claudia Schachtzabel, Gerd Wagner,

C. Christoph Schultz, Christian Sorg and Ralf G. M. Schlösser

\section{Background}

Alterations in the dopaminergic reward system, predominantly the striatum, constitute core characteristics of schizophrenia.

\begin{abstract}
Aims
Functional connectivity of the dorsal striatum during rewardrelated trial-and-error learning was investigated in 17 people with schizophrenia and 18 healthy volunteers and related to striatal grey matter volume and psychopathology.
\end{abstract}

\section{Method}

We used voxel-based morphometry and psychophysiological interaction to examine striatal volume and connectivity.

\section{Results}

A reduced functional connectivity between left striatum and temporo-occipital areas, precuneus and insula could be detected in the schizophrenia group. The positive correlation between grey matter volume and functional connectivity of the left striatum yielded significant results in a very similar network. Connectivity of the left striatum was negatively correlated with negative symptoms.

\section{Conclusions}

Present results suggest a disruption in striatal functional connectivity that is closely linked to grey matter morphometry of the striatum. Decreased connectivity between the striatum and psychopathologically relevant networks may explain the emergence of negative symptoms.

\section{Declaration of interest}

None.
Learning on the basis of feedback and reward has been shown to involve predominantly frontostriatal networks. One major part of these networks is the striatum, which integrates affective, motor and cognitive information and influences goal-directed behaviour. ${ }^{1}$ Several functional magnetic resonance imaging (fMRI) studies in healthy controls showed a strong bilateral activation of the striatum, mainly in its dorsal part during processing of reinforcement tasks. ${ }^{2}$ Apart from the striatum projection regions of the dopaminergic midbrain, such as medial frontal cortex, ${ }^{3}$ the amygdala-hippocampus complex ${ }^{4}$ and insula ${ }^{5}$ are critically involved in the processing of reinforcement and reward as well as in reward-based learning processes. Of note, midbrain dopaminergic networks have been shown to respond predominantly towards unpredicted rewards, i.e. when a so-called prediction error is committed. Alterations in the dopaminergic reward system, predominantly in frontostriatal networks, constitute core characteristics of the disorder of schizophrenia. ${ }^{6}$ Accordingly, patients have repeatedly been found to be impaired in processes involving this system, such as in reinforcement learning processes. For instance, in a previous study ${ }^{7}$ we showed that the ability to learn contingencies on the basis of rewardrelated feedback is altered on a behavioural as well as on a neuronal level in people with schizophrenia. Here, a hypoactivation of the dorsal striatum (i.e. putamen), dorsal cingulate and the superior frontal cortex was found in patients relative to healthy controls during processing of reinforcement and reward. Aberrant activation in the striatum, both in the ventral ${ }^{8,9}$ and dorsal $^{10,11}$ portion, has repeatedly been reported in people with schizophrenia in the context of reward processing. Looking at differences between patients treated with different therapy types, Juckel and colleagues showed a reduced activation in the ventral striatum during response to rewarding stimuli, compared with controls, in people with schizophrenia who were medicated ${ }^{12}$ and unmedicated. ${ }^{13}$ Schlagenhauf et al $(2008)^{14}$ found a significantly decreased activation of the ventral striatum in patients treated with typical antipsychotics compared with controls and patients treated with atypical antipsychotics during anticipation of rewarding stimuli. In addition to a disrupted activation in frontostriatal networks alterations in the so-called salience network comprising the insula and the anterior cingulate cortex have been reported in people with schizophrenia during processing of reinforcement and reinforcement-based learning. ${ }^{15-17}$ For instance, using a delayed incentive paradigm with monetary rewards Walter et $a l^{17}$ found disrupted activation in the anterior cingulate in patients, with only healthy controls showing increasing activation with increasing reward. These disruptions within the salience network have been associated with both positive and negative symptoms (i.e. passivity symptoms) of the disorder. ${ }^{18}$ An inappropriate allocation of salience to internal representations and external events, potentially as a phenomenological consequence of altered neuronal activation in the salience network, is being discussed as the underlying psychopathological mechanism.

In summary, the studies described above show strong evidence for altered activation in networks involved in the processing of reinforcement and reward in patients with schizophrenia. Surprisingly, barely any evidence exists with regard to the functional connectivity between or within these networks in association with reward processing in patients. The first evidence came from Schlagenhauf et al (2009) ${ }^{19}$ who found a reduced functional connectivity between two brain regions (i.e. ventral striatum and medial frontal cortex) known to be important predominantly in the context of reward delivery. Gradin et $a l^{20}$ found not only a reduced activation in, among others, putamen and nucleus accumbens but also a weaker functional connectivity between the dopamine-rich midbrain and the right insulaanterior cingulate cortex salience network that correlated with the severity of psychotic symptoms in patients with schizophrenia. Moreover, it has been hypothesised that structural alterations in regions with a high incidence of dopamine transporters such as the striatum may lead to altered activation and connectivity in the dopamine reward system as well as negative symptoms and passivity as their clinical manifestation. ${ }^{18}$ In accordance with this 
assumption, the striatum has been reported to be structurally altered in patients with schizophrenia. The majority of studies reported increases in putaminal volume, ${ }^{21-24}$ which have been linked to long-term antipsychotic treatment, ${ }^{25}$ whereas some studies also found decreased volumes ${ }^{26,27}$ or no volume alterations. ${ }^{28}$ Surprisingly, the direct association between structural alterations and functional activation or connectivity of the striatum has barely been investigated in individuals with schizophrenia. Against this background the present study aimed at investigating the potential association between functional connectivity and grey matter structure of the dorsal striatum (i.e. putamen) in patients with schizophrenia during processing of a reward-learning task and to relate potential alterations in connectivity to the degree of negative symptoms and blunted affect (passivity). We expected the connectivity of the dorsal striatum to relevant midbrain and salience network regions to be reduced in patients with schizophrenia, with this reduced connectivity being directly linked to structural characteristics of the striatum.

\section{Method}

\section{Participants}

The study sample consisted of 17 patients ( 9 male, 8 female) with a DSM-IV diagnosis of schizophrenia (schizophrenia group) and 18 controls (11 male, 7 female; control group ). ${ }^{29}$ All participants were right-handed. ${ }^{30}$ On average, the schizophrenia group were 33.1 years old (s.d. =7.9). Education was measured in years of schooling: German Abitur, 13 years; mittlere Reife, 10 years; Volksschule, 8 years. Mean education in the schizophrenia group was 11.5 years $($ s.d. $=1.9)$. In the control group the mean age was 29.3 (s.d.=6.3) with a mean education of 12.7 years $($ s.d. $=1.0)$. There was no significant difference between the groups in terms of age $(t(33)=-1.7)$ but a significant difference regarding education $(t(23.5)=2.2, P<0.04$, corrected for unequal variances). Diagnosis was established by the Structured Clinical Interview for DSM-IV Axis I Disorders (SCID-I) ${ }^{31}$ and confirmed by a clinical psychiatrist (Ch.S.). All participants in the schizophrenia group were free of any concurrent psychiatric diagnosis and had no neurological conditions. They were in remission from an acute psychotic episode. Thirteen were on stable medication with atypical antipsychotics, two were on stable medication with atypical antipsychotics and antidepressant medication (venlafaxine, citalopram) and received typical antipsychotic medication (haloperidol, flupenthixol). Antipsychotic doses were converted to chlorpromazine equivalents according to Woods. $^{32}$ Mean chlorpromazine equivalents were 544.4 (s.d.=293.3). The psychopathological status of the patients was assessed with the Positive and Negative Syndrome Scale (PANSS). ${ }^{33}$ Ratings were 16.1 (s.d.=4.4) on the positive subscale, 21.2 (s.d.=6.4) on the negative subscale and 37.0 (s.d.=8.0) on the general psychopathology scale.

The control group were screened by comprehensive assessment procedures for medical, neurological and psychiatric history. Exclusion criteria were current and potentially interfering medical conditions, any current or previous neurological or psychiatric disorder, and first-degree relatives with Axis I psychiatric or neurological disorders. All participants gave written informed consent to the study protocol. The protocol is in accordance with the ethical standards of the Declaration of Helsinki and was approved by the Ethics Committee of the Friedrich-Schiller-University Medical School.

\section{Experimental design}

Using the Presentation software package (Neurobehavioral Systems Inc. Albany, CA, USA; see http://www.neurobs.com) stimuli were projected onto a transparent screen inside the scanner tunnel, which could be viewed by the participant through a mirror system mounted on top of the MRI head coil. The participants' responses were recorded using an MRI-compatible fibre-optic response device (Lightwave Medical Industries, Canada) with four button keypads for the right hand. Participants were informed that they would be presented with a card with a geometrical figure on it (i.e. cross, half-moon, triangle or pentagon) and were asked to guess whether the figure on the card predicted a value higher or lower than the number five. Each figure predicted the respective value with a probability of either $50 \%$ or $100 \%$. Each correct guess was followed by a monetary reward $(+0.50 €)$ whereas each wrong guess was followed by a punishment $(-0.50 €)$. Participants were instructed that the figure predicted the respective value with a certain probability but were not informed about the predictive probabilities of the respective figures. The whole paradigm consisted of a series of 64 interleaved trials with 32 trials for each probability condition distributed across the whole task sequence. Each trial started with the presentation of the probability condition-specific figure, which was shown for $1.5 \mathrm{~s}$. After an interstimulus interval lasting $4.5 \mathrm{~s}$ a question mark was presented for $2.5 \mathrm{~s}$ during which participants had to answer by a button press. After another interstimulus interval of $4.5 \mathrm{~s}$ the correct solution followed by the indication of a reward or punishment appeared for $2.5 \mathrm{~s}$. Participants were compensated according to their performance, although a minimum of $€ 20$ was guaranteed for volunteering. Each trial ended with an intertrial interval lasting $3.5 \mathrm{~s}$. In addition, we introduced a temporal jitter by varying the second interstimulus interval between 4.5 and $5.5 \mathrm{~s}$ in order to increase sensitivity.

\section{fMRI procedure}

Functional data were collected on a $3 \mathrm{~T}$ whole-body system equipped with a 12-element receive-only head matrix coil (MAGNETOM TIM Trio, Siemens). Foam pads were used for positioning and immobilisation of the participant's head within the head coil. $\mathrm{T}_{2}{ }^{*}$-weighted images were obtained using a gradient-echo echo-planar imaging (EPI) sequence (repetition time $(\mathrm{TR})=2040 \mathrm{~ms}$; echo time $(\mathrm{TE})=26 \mathrm{~ms}$; flip angle, $90^{\circ}$ ) with 40 contiguous transverse slices of $3.3 \mathrm{~mm}$ thickness covering the entire brain. Matrix size was $72 \times 72$ pixels with in-plane resolution of $2.67 \times 2.67 \mathrm{~mm}$ corresponding to a field of view of $192 \times 192 \mathrm{~mm}$. A series of 645 whole-brain volume sets were acquired, with the first three images of each series being discarded. High-resolution anatomical $\mathrm{T}_{1}$-weighted volume scans (MP-RAGE) were obtained in sagittal orientation $(\mathrm{TR}=2300 \mathrm{~ms}$; $\mathrm{TE}=3.03 \mathrm{~ms}$; inversion time $(\mathrm{TI})=900 \mathrm{~ms}$; flip angle, 9; filed of view $($ FOV $)=256 \mathrm{~mm}$; matrix $256 \times 256$; number of sagittal slices, 192; acceleration factor $(\mathrm{PAT})=2$; acquisition time $(\mathrm{TA})=5: 21 \mathrm{~min})$ with an isotropic resolution of $(1 \times 1 \times 1) \mathrm{mm}^{3}$.

\section{Data analysis}

\section{Behavioural data}

Performance was assessed by the percentage of correct reactions in each probability condition. A two-sample $t$-test for each probability condition $(50 \%, 100 \%)$ was performed to test for differences between the groups. In addition we applied the concept of temporal difference learning to estimate an individual learning rate parameter ${ }^{34}$ (for analysis details see Koch et $a l^{7}$ ). Here, the change in associative strength of stimulus $i$ on each trial $j,\left(\Delta V_{\mathrm{ij}}\right)$, was determined as: 


$$
\Delta V_{i j}= \begin{cases}\gamma_{i j} \times A_{i j} & j=1 \\ \gamma_{i j} \times\left(A_{i j}-\sum_{k=1}^{j-1} \Delta V_{i k}\right) & j>1\end{cases}
$$

In psychological terms, $\gamma_{i j}$ can be interpreted as stimulus-reward associability $^{35}$ and moreover as a discount factor determining the extent to which rewards that arrive earlier are more important for learning than rewards that arrive later. ${ }^{5,36}$ In case of a correct guess and monetary gain, $A_{i j}$ of a trial takes the value 1 , for nonreinforcement trials (i.e. incorrect guess and monetary loss) $A_{i j}$ was coded by $0 . \sum_{k=1}^{j-1} \Delta V_{i k}$ illustrates the expected reward or associative strength of each trial $j$. The learning rate (LR), modelled to assess the individual learning capability under stable learning conditions (i.e. $100 \%$ condition), was then calculated as follows:

$$
L R_{i}=1-\frac{1}{16} \times \sum_{j=1}^{16}\left(V_{i j / \max }-\Delta V_{i j}\right)
$$

where $V_{i j / \max }$ reflects the expected reward in case of optimal learning performance. Given 16 trials for each stimulus category $1-\frac{1}{16}$ gives the mean LR for each condition. Thus, higher learning rate values stand for better learning. A two-sample $t$-test served for comparing the individual learning rates between the groups. A repeated-measures ANOVA with associative strength (i.e. associative strength values across the 16 trials) as within-participant factor, and group (schizophrenia, control) as between-participant factor served for testing for group differences across the learning process under stable learning conditions. To investigate whether the learning performance was associated with the degree of negative symptoms, a partial correlation between individual learning rate and degree of negative symptoms (corrected for the influence of general psychopathology) was conducted.

\section{fMRI data}

Preprocessing and statistical analysis of the fMRI data was performed using SPM8 (http://www.fil.ion.ucl.ac.uk/spm). Functional data were corrected for differences in time of acquisition by sinc interpolation, realigned to the first image of the session and linearly and non-linearly normalised to the Montreal Neurological Institute (MNI) reference brain (MNI 152). Data were spatially smoothed with a Gaussian kernel $(8 \mathrm{~mm}$, full-width at half maximum (FWHM)) and high-pass filtered with a $128 \mathrm{~s}$ cut-off. All data were inspected for movement artefacts. Participants with movement parameters exceeding $3 \mathrm{~mm}$ translation on the $x$-, $y$-, or $z$-axis or 3 rotation were excluded ( 1 patient and 1 healthy control, which resulted in a final sample size of 17 patients and 18 controls). In addition, individual movement parameters entered the analyses as covariates of no interest.

On the first level, brain activations were then analysed voxelwise to calculate statistical parametric maps of $t$-statistics for the $50 \%$ probability condition (i.e. activation during responding to triangles and pentagons), the $100 \%$ probability condition (i.e. activation during responding to half-moons and crosses) and positive compared with negative reinforcement (i.e. activation during presentation of monetary win $v$. monetary loss).

Blood oxygenation level-dependent (BOLD) signal changes for the different conditions were modelled as a covariate of variable length boxcar functions and convolved with a canonical haemodynamic response function. These haemodynamic response function were then used as individual regressors within the general linear model.

\section{Psychophysiological interactions}

We used psychophysiological interaction (PPI) analysis to investigate our hypotheses of altered striatal (i.e. putamen) connectivity in the schizophrenia group in association with reward processing. The PPI analysis is based on bilinear interaction terms. ${ }^{37}$ Analogous to the use of modulatory bilinear terms in systems engineering the interaction term can be regarded as an expression of the modulatory input of an external factor or signal on the interaction between a target and a source region. Against the background of our hypothesis of an aberrant striatal connectivity in people with schizophrenia, we identified striatal seed regions of interest based on the peak activation from the second-level analysis (i.e. positive $v$. negative feedback) in the schizophrenia group (left putamen activation maximum: $x=-14, y=10$, $z=-8$; right putamen activation maximum: $x=12, y=6$, $z=-7$ ) and the control group (left putamen activation maximum: $x=-10, y=4, z=-12$; right putamen activation maximum: $x=8, y=6, z=-10)$. Next, individual time series from spheres with $4 \mathrm{~mm}$ radius around the individual activation maximum next to the group-specific striatal seed region were extracted for each participant. These individual spheres were all located in the putamen with the distance between individual centre coordinates and centre coordinates of seed regions (as described above) not exceeding a radius of $10 \mathrm{~mm}$ for all participants (apart from one healthy control where peak coordinates of left and right putamen were located at $x=-24, y=2, z=-4$ and $x=29, y=0, z=5$ and another healthy control where peak coordinates of right putamen were located at $x=22, y=4, z=-10)$. These time series constituted the physiological component of the PPI. The contrast between positive $v$. negative feedback constituted the psychological component. The interaction (i.e. the element-by-element product) between the psychological component and the physiological component was used as the PPI regressor.

With this implementation of the PPI analysis, significant activations of a particular area would reflect increased functional connectivity between the source area (i.e. left/right putamen) and the activated regions during processing of positive $v$. negative feedback. One-way ANOVAs served for illustrating regions functionally connected with the striatal seed regions in both the groups as well as in the control group compared with the schizophrenia group. To investigate whether differences in connectivity between the groups are as a result of differences in behavioural performance, we performed additional one-way ANCOVAs with performance (i.e. the learning rate) as covariate. To correct for false positive errors, the double-threshold approach was used, which imposes both an activation threshold and a cluster size threshold. Activation clusters were considered significant if they reached a threshold of $P<0.001$ on the voxellevel and exceeded an extent of 20 voxels on the cluster-level, which is equivalent to a threshold of $P=0.05$, corrected. In addition, we performed small volume corrections (based on $4 \mathrm{~mm}$ spheres around maximum-activated voxels) for all significant results of the group comparisons.

\section{Voxel-based morphometry - PPI}

To investigate whether alterations in grey matter structure (i.e. grey matter volume) in patients with schizophrenia underlie altered functional connectivity we performed voxel-based morphometry (VBM) applying the VBM-toolbox (http:// dbm.neuro.uni-jena.de/) implemented in SPM8 using default parameters. Images were bias-corrected, tissue classified, and linearly (i.e. 12-parameter affine registration) and non-linearly (i.e. warping regularisation) registered. Subsequently, grey matter and white matter segments were modulated by multiplication with 
the non-linear components derived from the normalisation matrix in order to preserve actual grey matter and white matter values locally. Images were smoothed with a Gaussian kernel of $8 \mathrm{~mm}$ FWHM). Finally, to investigate the hypothesised association between altered striatal connectivity and grey matter structure patients' age-corrected grey matter values were extracted from the left and right putamen (i.e. $4 \mathrm{~mm}$ sphere at $x=9, y=6$, $z=-11$ and $x=-14, y=11, z=-8)$ and correlated with taskrelated functional connectivity of the left and right putamen (i.e. seed regions as described above). As before, thresholding was performed according to the double-threshold approach, i.e. activation clusters were considered significant if they reached or exceeded an extent of 20 voxels, which is equivalent to a threshold of $P=0.05$, corrected. Potential group differences in putaminal grey matter volume (i.e. extracted grey matter values from left and right putamen) were investigated by two-sample $t$-tests. Although the reliability of chlorpromazine equivalents is not uncontroversial, ${ }^{38}$ extracted grey matter values from left and right putamen were correlated with chlorpromazine equivalents ${ }^{32}$ to explore the effects of antipsychotic medication on putamen volume.

\section{Psychopathology - PPI}

Finally, to investigate the hypothesised association between altered striatal connectivity and negative symptoms negative PANSS scores and passivity scores (i.e. N1 blunted affect) were correlated with task-related functional connectivity of the left and right putamen (i.e. seed regions as described above). To adjust for the confounding effects of general psychopathology PANSS general scores were added as a covariate-of-no-interest. Again, thresholding was based on the double-threshold approach, i.e. correlation between functional connectivity and psychopathology was considered significant if clusters reached or exceeded an extent of 20 voxels corresponding to a threshold of $P=0.05$, corrected.

\section{Results}

\section{Behavioural data}

In the $50 \%$ condition where prediction or learning was impossible both the schizophrenia and the control group showed a comparable percentage of correct responses (controls: 50.3 (s.d. $=11.4$ ), patients: 46.5 (s.d. $=8.8), t(33)=1.1$, not significant). In the $100 \%$ condition the schizophrenia group showed a lower percentage of correct responses compared with controls (control group: 88.7 (s.d.=15.9), schizophrenia group: 78.7 (s.d.=13.7), $t(33)=2.0$, $P<0.054)$. The assessment of the individual learning capability under stable learning conditions yielded a mean learning rate of 0.8 (s.d. $=0.1$ ) in the schizophrenia group and $0.9($ s.d. $=0.2)$ in the control group. The independent two-sample $t$-test testing for differences in the individual learning rates between the groups yielded a trend significance $(t(33)=1.9, P<0.063)$. The ANOVA testing for group differences in associative strength across the learning process under stable learning conditions yielded a trend significance for group $(F(1,33)=3.7, P<0.063)$, a main effect of associative strength $(F(1,37)=49.9, P<0.001)$ and a borderline significant interaction $(F(15,33)=1.7, P=0.057)$ indicating lower overall performance as well as a worse learning performance across time in the schizophrenia group compared with the control group.

\section{PPI}

The one-way ANOVA investigating functional connectivity of the left putamen in association with processing of positive $v$. negative feedback yielded a network consisting of mainly bilateral occipital and frontal regions, the left caudate and the right insula in the control group (Table 1, online Fig. DS1) and a network comprising predominantly bilateral occipital and frontal regions and the left temporal cortex in the schizophrenia group (Table 1, online Fig. DS1).

The one-way ANOVA investigating functional connectivity of the right putamen in association with processing of positive $v$. negative feedback yielded a network consisting of mainly bilateral occipital, frontal and temporal regions, the right caudate and the insula bilaterally in the control group (Table 2) and a network comprising predominantly bilateral occipital and frontal regions in the schizophrenia group (Table 3).

The one-way ANOVA comparing functional connectivity of the left putamen between the groups yielded a significantly lower connectivity in the schizophrenia group in a network containing the right middle temporal gyrus, the occipital lobe bilaterally, the right insula and the precuneus (Table 4, online Fig. DS1). The one-way ANCOVA comparing functional connectivity of the left putamen between the groups corrected for performance (i.e. learning rate) yielded a significantly lower connectivity in the schizophrenia group in a network containing the right middle temporal gyrus, the occipital lobe bilaterally and the right insula (Table 4). The opposite contrast showed no significantly increased connectivity in the control group, neither with nor without correcting for performance.

The one-way ANOVA comparing functional connectivity of the right putamen between the groups yielded a significantly lower connectivity in the schizophrenia group in the left precuneus $(x=-30, y=-74, z=40$, Brodmann's area (BA) 19, $k=69$, $T=4.40)$. The one-way ANCOVA comparing functional connectivity of the right putamen between the groups corrected for performance (i.e. learning rate) yielded no significant results. The opposite contrast showed a significantly increased connectivity in the schizophrenia group in the left inferior frontal gyrus/BA 45 (ANOVA: $x=50, y=16, z=4, k=18, T=4.88$, ANCOVA corrected for performance: $x=50, y=16, z=4, k=53$, $T=5.82$ ). Finally, all regions showing significant group differences remained significant when analysed with a small volume correction (i.e. $P<0.006$ or smaller).

\section{VBM - PPI}

The positive correlation between grey matter volume of the left putamen and functional connectivity of the left putamen seed region yielded significant results in a network containing mainly the right middle temporal gyrus, the occipital lobe bilaterally, the left insula, the left precuneus and the frontal cortex bilaterally (Table 5, online Fig. DS2). The negative correlation yielded no significant effects. There were also no significant effects for the right putamen. The two-sample $t$-test comparing grey matter volumes between the groups yielded a significant result both for the left (schizophrenia group: 0.56 (s.d.=0.07), control group: 0.64 (s.d. $=0.06), t(33)=-3.4, P<0.002)$ and the right (schizophrenia group 0.61 (s.d. $=0.06)$, control group: 0.68 (s.d. $=0.05)$, $t(33)=-3.3, \quad P<0.003)$ putamen. There was no significant correlation between chlorpromazine equivalent dosage and volume of the right or left putamen.

\section{Psychopathology - PPI}

The positive correlation between degree of negative symptoms and functional connectivity of the left putamen seed region yielded a small cluster in the left cerebellum $(x=-22, y=-40, z=-32$, $k=20, T=4.51)$. The negative correlation yielded significant results in a network comprising the occipital lobe bilaterally, the right insula, the anterior cingulate and the frontal cortex 
Table 1 Montreal Neurological Institute coordinates of activation maxima (SPM $\{T\}$ value, $k=$ number of voxels in cluster) for task-related functional connectivity (positive $v$. negative feedback) in the control group and schizophrenia group with the left putamen as seed region ${ }^{\mathrm{a}}$

\begin{tabular}{|c|c|c|c|c|c|}
\hline & Side & Brodmann's area & k & $T$ & $x, y, z$ \\
\hline \multicolumn{6}{|l|}{ Control group } \\
\hline Occipital lobe, superior parietal lobe, cerebellum & Left/right & 18 & 29390 & 9.25 & $-30,-86,-10$ \\
\hline Anterior insula, middle frontal gyrus & Right & 13 & 2547 & 5.92 & $42,14,16$ \\
\hline Caudate & Left & & 178 & 5.89 & $-10,12,-6$ \\
\hline Frontal lobe, medial frontal gyrus & & 9 & 781 & 5.76 & $6,40,30$ \\
\hline Frontal lobe, inferior frontal gyrus & Left & 47 & 66 & 5.39 & $-28,16,-20$ \\
\hline Frontal lobe, middle frontal gyrus & Left & 9 & 489 & 5.06 & $-42,10,30$ \\
\hline Frontal lobe, middle frontal gyrus & Right & 8 & 215 & 4.6 & $26,26,46$ \\
\hline Frontal lobe, precentral gyrus & Right & 6 & 171 & 4.36 & $40,-12,36$ \\
\hline Cingulate gyrus & & 24 & 27 & 4.12 & $4,-4,34$ \\
\hline Frontal lobe, precentral gyrus & Left & 4 & 43 & 4.09 & $-62,-12,30$ \\
\hline Cingulate gyrus & Left & 31 & 22 & 3.92 & $-24,-34,38$ \\
\hline Frontal lobe, middle frontal gyrus & Left & 6 & 46 & 3.87 & $-30,14,48$ \\
\hline Parahippocampal gyrus & Left & 34 & 22 & 3.82 & $-14,-4,-14$ \\
\hline Frontal lobe, inferior frontal gyrus & Left & 47 & 24 & 3.77 & $-34,26,-4$ \\
\hline \multicolumn{6}{|l|}{ Schizophrenia group } \\
\hline Frontal lobe, inferior frontal gyrus & Left & 47 & 225 & 7.33 & $-28,16,-18$ \\
\hline Temporal lobe, fusiform gyrus & Right & 37 & 1222 & 7.11 & $42,-54,-16$ \\
\hline Temporo-occipito-parietal lobe, precuneus & Left & 31 & 2374 & 6.45 & $-24,-72,26$ \\
\hline Occipital lobe, middle occipital gyrus & Right & 19 & 765 & 5.25 & $38,-82,16$ \\
\hline Frontal lobe, medial frontal gyrus & Right & 9 & 129 & 5.11 & $6,42,20$ \\
\hline Temporal lobe, superior temporal gyrus & Left & 22 & 161 & 4.96 & $-52,-40,6$ \\
\hline Frontal lobe, inferior frontal gyrus & Right & 47 & 56 & 4.77 & $26,12,-16$ \\
\hline Frontal lobe, middle frontal gyrus & Right & 46 & 47 & 4.66 & $54,26,24$ \\
\hline Parahippocampal gyrus & Left & 34 & 59 & 4.58 & $-14,0,-14$ \\
\hline Frontal lobe, medial frontal gyrus & & 9 & 80 & 4.11 & $2,50,38$ \\
\hline Parahippocampal gyrus & Right & 34 & 27 & 4.08 & $16,0,-12$ \\
\hline Frontal lobe, inferior frontal gyrus & Right & 9 & 140 & 3.95 & $42,8,30$ \\
\hline
\end{tabular}

\begin{tabular}{|c|c|c|c|c|c|}
\hline & Side & Brodmann's area & $k$ & $T$ & $x, y, z$ \\
\hline Occipital lobe & Left/right & 18/19 & 17132 & 8 & $-20,-84,-6$ \\
\hline Anterior insula & Right & 13 & 375 & 6.68 & $36,24,0$ \\
\hline Frontal lobe, medial frontal gyrus & Right & 6 & 1095 & 6.47 & $6,40,34$ \\
\hline Parietal lobe, postcentral gyrus & Right & 40 & 124 & 5.83 & $56,-30,50$ \\
\hline Frontal lobe, middle frontal gyrus & Right & 46 & 485 & 5.53 & $46,28,24$ \\
\hline Frontal lobe, middle frontal gyrus & Left & 6 & 125 & 5.41 & $-34,14,54$ \\
\hline Temporal lobe, middle temporal gyrus & Left & 22 & 144 & 5.16 & $-52,-38,4$ \\
\hline Cingulate gyrus & Left & 31 & 201 & 4.79 & $-12,-40,40$ \\
\hline Frontal lobe, middle frontal gyrus & Right & 9 & 290 & 4.75 & $40,10,40$ \\
\hline Caudate & Right & & 347 & 4.7 & $8,14,-2$ \\
\hline Frontal lobe, inferior frontal gyrus & Left & 9 & 490 & 4.69 & $-50,16,24$ \\
\hline Anterior insula & Left & 13 & 146 & 4.64 & $-36,22,-6$ \\
\hline Cerebellum & Left & & 54 & 4.6 & $-8,-56,-18$ \\
\hline Frontal lobe, precentral gyrus & Left & 4 & 61 & 4.43 & $-46,-10,46$ \\
\hline Temporal lobe, middle temporal gyrus & Right & 39 & 41 & 4.31 & $42,-64,24$ \\
\hline Parietal lobe, postcentral gyrus & Left & 40 & 44 & 4.29 & $-52,-38,44$ \\
\hline Frontal lobe, middle frontal gyrus & Right & 6 & 59 & 4.27 & $32,2,48$ \\
\hline Posterior cingulate & Right & 30 & 36 & 4.21 & $6,-48,18$ \\
\hline Parahippocampal gyrus & Left & 35 & 35 & 4.19 & $-16,-24,-8$ \\
\hline Frontal lobe, middle frontal gyrus & Right & 10 & 44 & 4.19 & $42,56,8$ \\
\hline Temporal lobe, superior temporal gyrus & Right & 39 & 122 & 4.11 & $50,-50,28$ \\
\hline Frontal lobe, superior frontal gyrus & Right & 10 & 26 & 4.01 & $26,52,0$ \\
\hline Frontal lobe, middle frontal gyrus & Left & 6 & 35 & 3.93 & $-34,4,60$ \\
\hline Substantia nigra & Right & & 39 & 3.83 & $8,-14,-8$ \\
\hline Frontal lobe, paracentral lobule & Left & 6 & 23 & 3.81 & $-4,-32,58$ \\
\hline
\end{tabular}


Table 3 Montreal Neurological Institute coordinates of activation maxima (SPM $\{T\}$ value, $k=$ number of voxels in cluster) for taskrelated functional connectivity (positive $v$. negative feedback) in the schizophrenia group with the right putamen as seed region ${ }^{a}$

\begin{tabular}{|lccccc|}
\hline & Side & Brodmann's area & $k$ & $T$ & \multicolumn{1}{c}{$\boldsymbol{T}, y, z$} \\
\hline Frontal lobe, precentral gyrus & Right & 44 & 28 & 5.16 & $50,16,4$ \\
\hline Frontal lobe, subcallosal gyrus & Left & 34 & 31 & 5.12 & $-14,0,-10$ \\
\hline Occipital lobe & Right & 19 & 393 & 4.75 & $32,-72,28$ \\
\hline Cerebellum & Right & & 93 & 4.7 & $40,-48,-20$ \\
\hline Frontal lobe, inferior frontal gyrus & Right & 9 & 150 & 4.66 & $44,6,30$ \\
\hline Occipital lobe, lingual gyrus & Left & 18 & 368 & 4.5 & $-26,-74,-8$ \\
\hline Occipital lobe, cuneus & Left & 18 & 33 & 4.18 & $-26,-72,26$ \\
\hline Cerebellum & Right & & 180 & 4.16 & $32,-72,-12$ \\
\hline Occipital lobe, middle occipital gyrus & Left & 18 & 66 & 4.09 & $-22,-96,8$ \\
\hline Frontal lobe, superior frontal gyrus & Left & 6 & 21 & 4.03 & $-8,20,56$ \\
\hline Occipital lobe, lingual gyrus & Right & 17 & 44 & 4.01 & $16,-94,-6$ \\
\hline Frontal lobe, middle frontal gyrus & Right & 46 & 52 & 3.94 & $54,30,24$ \\
\hline Frontal lobe, middle frontal gyrus & Left & 6 & 33 & 3.79 & $-38,2,54$ \\
\hline Frontal lobe, medial frontal gyrus & Right & 6 & 22 & 3.79 & $4,42,36$ \\
\hline a. One-way ANova at $P<0.05$, corrected. & & & & & \\
\hline
\end{tabular}

Table 4 Montreal Neurological Institute coordinates of activation maxima (SPM $\{T\}$ value, $k=$ number of voxels in cluster) for weaker task-related functional connectivity (positive $v$. negative feedback) in the schizophrenia group compared with the control group with the left putamen as seed region with and without correction for performance (i.e. learning rate) ${ }^{\mathrm{a}}$

\begin{tabular}{|c|c|c|c|c|c|}
\hline & Side & Brodmann's area & k & $T$ & $x, y, z$ \\
\hline \multicolumn{6}{|l|}{ Uncorrected for performance } \\
\hline Temporal lobe, middle temporal gyrus & Right & 22 & 43 & 4.65 & $48,-46,0$ \\
\hline Occipital lobe, fusiform gyrus & Left & 18 & 35 & 4.28 & $-24,-96,-10$ \\
\hline Anterior insula ${ }^{\mathrm{b}}$ & Right & 13 & 19 & 4.05 & $30,4,14$ \\
\hline Parietal lobe, precuneus & Left & 7 & 21 & 3.94 & $-14,-60,40$ \\
\hline Occipital lobe, inferior occipital gyrus & Right & 18 & 31 & 3.94 & $34,-90,-10$ \\
\hline Parietal lobe, precuneus & & 7 & 48 & 3.78 & $0,-50,44$ \\
\hline \multicolumn{6}{|l|}{ Corrected for performance } \\
\hline Temporal lobe, middle temporal gyrus & Right & 22 & 21 & 4.20 & $48,-46,0$ \\
\hline Occipital lobe, fusiform gyrus & Left & 18 & 38 & 4.49 & $-24,-94,-10$ \\
\hline Anterior insula & Right & 13 & 23 & 4.41 & $28,4,16$ \\
\hline Occipital lobe, lingual gyrus & Right & 19 & 53 & 3.87 & $14,-48,-4$ \\
\hline
\end{tabular}

\begin{tabular}{|c|c|c|c|c|c|}
\hline & Side & Brodmann's area & $k$ & $T$ & $x, y, z$ \\
\hline Parietal lobe, precuneus & Left & 19 & 79 & 8.61 & $-20,-82,40$ \\
\hline Cerebellum & Right & & 154 & 7.11 & $4,-68,-24$ \\
\hline Frontal lobe, superior frontal gyrus & Right & 6 & 32 & 5.42 & $4,30,58$ \\
\hline Frontal lobe, medial frontal gyrus & Left & 9 & 56 & 5.37 & $-16,40,14$ \\
\hline Temporal lobe, middle temporal gyrus ${ }^{b}$ & Right & 41 & 16 & 5.25 & $42,-38,16$ \\
\hline Frontal lobe, superior frontal gyrus & Left & 6 & 77 & 5.21 & $-2,2,66$ \\
\hline Inferior parietal lobe & Left & 40 & 71 & 5.14 & $-64,-32,30$ \\
\hline Posterior cingulate & Right & 31 & 79 & 5.1 & $24,-64,14$ \\
\hline Frontal lobe, precentral gyrus & Left & 6 & 151 & 4.99 & $-42,-4,54$ \\
\hline Occipital lobe, cuneus & Right & 19 & 77 & 4.92 & $14,-80,38$ \\
\hline Middle insula & Left & 13 & 23 & 4.73 & $-42,-10,24$ \\
\hline Parietal lobe, postcentral gyrus & Left & 3 & 42 & 4.67 & $-34,-30,66$ \\
\hline Frontal lobe, precentral gyrus & Left & 6 & 23 & 4.18 & $-52,0,44$ \\
\hline
\end{tabular}


bilaterally (Table 6). Similar results were detectable for the correlation with passivity (i.e. N1 blunted affect). The positive correlation between degree of negative symptoms and functional connectivity of the right putamen seed region yielded no significant results. The same applied to the negative correlation with passivity symptoms. The negative correlation showed significant activation in the anterior cingulate and the middle frontal gyrus bilaterally for the degree of negative symptoms (Table 6) and in the left insula and bilateral middle frontal gyrus for the degree of passivity.

\section{Discussion}

\section{Performance}

Present results indicate that the schizophrenia group showed a worse performance in reward-related probabilistic trial-and-error learning as compared with the healthy control group. This was indicated by differences in percentage of correct responses as well as learning rates under fully predictable learning conditions. Results are roughly in line with previous studies revealing impaired performance for reward-related learning in schizophrenia. ${ }^{7,39}$ It should be noted, however, that not only the magnitude of impairment in our previous study was larger, most likely because of a more difficult version of the task that was based on three instead of only two learning conditions, but also that results in the present study showed only a trend towards significance. As expected, for the condition under which learning was not possible (i.e. $50 \%$ probability), both groups revealed a similar percentage of correct responses with no significant differences between groups.

\section{Functional connectivity}

In the control group, within-group analysis revealed processing of positive reinforcement in terms of monetary reward to be associated with an increased connectivity between dorsal striatum (i.e. putamen) and fronto-occipital areas as well as caudate and anterior insula. In contrast, in the schizophrenia group, an increase in connectivity was mainly restricted to fronto-occipital areas. Comparison between groups revealed a reduced connectivity in the schizophrenia group between dorsal striatum and temporooccipital areas as well as precuneus and insula. The findings suggest that a deficient interplay between the striatum and the outlined networks may be a mechanism underlying the accumulating evidence of reduced striatal activation in association with reward processing in people with schizophrenia..$^{8-12,14,19,40}$ The results confirm recent initial findings of reduced functional connectivity in individuals with schizophrenia in the context of reward processing. In these studies, however, partly different networks were affected by reduced connectivity, namely ventral striatum and medial frontal cortex networks ${ }^{19}$ as well as midbrain and the insula-anterior cingulate cortex salience network. ${ }^{10}$ Our results suggest that a reduced connectivity between dorsal striatum and temporo-occipital as well as insular areas may be of relevance. There is mounting evidence showing that it is predominantly the dorsal part of the striatum which is critically involved in response-related functions.

This evidence is supported by the fact that there are strong anatomical connections between the putamen and primary, premotor and supplementary motor cortices. ${ }^{41}$ Studies illustrating putamen activity in association with reward delivery and response towards receipt of reward are in line with the putative relevance of the putamen in association with behavioural response towards reward delivery. As indicated by earlier findings the ventral striatum, on the other hand, may be predominantly involved in the anticipation of rewards not necessarily going along with a direct behavioural response. ${ }^{42}$ Thus, our results showing a decreased connectivity between dorsal striatum and temporo-occipital as well as insular areas in people with schizophrenia in association with reward presentation and response towards it, fit the picture

Table 6 Montreal Neurological Institute coordinates of activation maxima (SPM $\{T\}$ value, $k=$ number of voxels in cluster) for

the schizophrenia group's negative correlation between psychopathology (i.e. negative symptoms, blunted affect) and task-

related functional connectivity (positive $v$. negative feedback) of the left and right putamen ${ }^{\text {a }}$

\begin{tabular}{|c|c|c|c|c|c|}
\hline & Side & Brodmann's area & k & $T$ & $x, y, z$ \\
\hline \multicolumn{6}{|l|}{ Left putamen } \\
\hline \multicolumn{6}{|l|}{ Negative symptoms } \\
\hline Anterior cingulate & Left & 32 & 147 & 6.67 & $12,42,-2$ \\
\hline Anterior insula, inferior frontal gyrus & Right & $13 / 47$ & 112 & 5.95 & $32,20,-6$ \\
\hline Occipital lobe, lingual gyrus & Right & 18 & 194 & 5.52 & $18,-78,0$ \\
\hline Frontal lobe, medial frontal gyrus & Left & 10 & 33 & 5.0 & $-18,48,-4$ \\
\hline Anterior cingulate & Right & 24 & 81 & 4.92 & $-8,36,4$ \\
\hline Occipital lobe, inferior occipital gyrus & Left & 18 & 34 & 4.83 & $42,-84,-2$ \\
\hline \multicolumn{6}{|l|}{ Blunted affect } \\
\hline Anterior cingulate & Right & 32 & 31 & 5.85 & $12,42,-2$ \\
\hline Posterior cingulate & Right & 31 & 51 & 5.23 & $8,-22,40$ \\
\hline Occipital lobe, inferior occipital gyrus & Left & 18 & 34 & 5.18 & $-38,-86,-4$ \\
\hline Anterior insula, inferior frontal gyrus & Right & $13 / 47$ & 36 & 5.14 & $38,16,-8$ \\
\hline Anterior cingulate & Left & 24 & 112 & 4.96 & $-12,34,4$ \\
\hline Frontal lobe, superior frontal gyrus & Right & 10 & 20 & 4.95 & $24,54,0$ \\
\hline Occipital lobe, inferior occipital gyrus & Right & 18 & 60 & 4.71 & $26,-84,-2$ \\
\hline \multicolumn{6}{|l|}{ Right putamen } \\
\hline \multicolumn{6}{|l|}{ Negative symptoms } \\
\hline Anterior cingulate & Left & 24 & 147 & 6.67 & $-2,36,8$ \\
\hline Frontal lobe, middle frontal gyrus & Right & 9 & 112 & 5.95 & $34,26,34$ \\
\hline Anterior cingulate & Right & 24 & 194 & 5.52 & $12,34,4$ \\
\hline Frontal lobe, middle frontal gyrus & Left & 9 & 33 & 5.0 & $-36,20,30$ \\
\hline \multicolumn{6}{|l|}{ Blunted affect } \\
\hline Insula, inferior frontal gyrus & Left & 47 & 53 & 6.34 & $-28,24,4$ \\
\hline Frontal lobe, middle frontal gyrus & Left & 9 & 40 & 6.08 & $-36,20,28$ \\
\hline Frontal lobe, middle frontal gyrus & Right & 9 & 48 & 5.23 & $34,28,36$ \\
\hline
\end{tabular}


and indicate that a disruption within this behaviourally relevant dorsal striatal loop may constitute the basis for altered reward learning in people with schizophrenia.

Anatomically, vast connections between insula and dorsal as well as ventral portions of the striatum have been revealed in non-human primates and humans alike. ${ }^{43}$ Converging evidence from resting state fMRI (rs-fMRI) in humans displayed networks linking insular and putamen. ${ }^{44}$ Functionally, the insula plays a role for a broad range of tasks as diverse as emotional processing, interoception, self-recognition, perceptual decision-making and others. A large body of evidence points towards a functional subdivision of insula in an anterior region, mainly engaged in emotional processing and interoception and a posterior region mainly engaged in multimodal sensory processing. ${ }^{45}$ In a recent meta-analysis, Duerden et $a l^{46}$ examined data from more than 140 experiments reporting insula activation connected to emotional processing. Highest activation was found in bilateral anterior insula, regardless of emotional valence. The decrease in functional connectivity between striatum and anterior insula found in the schizophrenia group in the present study may thus affect the responsivity to affective stimulation normally caused by positive reinforcement or reward and may explain characteristic symptoms of the disorder such as flat affect or lack of motivation.

Apart from extensive connections to the insula the putamen has strong connections to the precuneus as demonstrated in monkey studies. ${ }^{47}$ Functionally, the precuneus has been linked to a variety of cognitive functions, such as episodic memoryretrieval, visuospatial imagery, consciousness and self-processing. A decrease in connectivity between striatum and precuneus may be a substrate of impaired cognitive processing. This assumption could be corroborated by correcting the connectivity differences for performance. After removing the influence of performance, the decreased connectivity between striatum and precuneus was no longer detectable. Taken together, results suggest that the impaired connectivity between striatum and anterior insula (mainly subserving functions such as emotion processing) as well as between striatum and precuneus (involved in cognitive processing) may have a direct influence on reinforcement-based learning as both, emotional and cognitive processing, are fundamental components of this kind of learning.

\section{Association between connectivity and grey matter structure}

A positive correlation between putamen grey matter volume and connectivity (i.e. the lower the grey matter volume, the lower the connectivity between putamen and fronto-occipital areas, precuneus as well as insula) was found in the schizophrenia group in largely those regions showing a decreased striatal connectivity in this group relative to the control group (see Fig. DS2). Hence, present results suggest that a disrupted functional interplay or connectivity between putamen and fronto-occipital areas, precuneus and insula is directly linked or may even constitute the consequence of an alteration in putaminal grey matter structure.

The results thus corroborate first evidence showing an association between functional connectivity and grey or white matter structural connectivity in partly overlapping regions in people with schizophrenia. As indicated by the group comparison of grey matter values extracted from the striatal seed region individuals with schizophrenia in the present study had significantly lower putaminal grey matter volumes relative to the control group. Previous studies on structural alterations of putamen are rather heterogeneous. The majority of studies reported increases in putaminal volume, ${ }^{21-24}$ which have been linked to long-term antipsychotic treatment ${ }^{25}$ whereas some studies also found decreased volumes ${ }^{26,27}$ or no volume alterations. ${ }^{28} \mathrm{~A}$ recent meta-analysis of 77 studies on schizophrenia, ${ }^{48}$ in which most of the included patients were treated with atypical antipsychotics, revealed no significant effect of antipsychotic treatment on global grey volume. Although we found no correlation between chlorpromazine equivalent dosage and striatal grey matter volume, effects of (previous) long-term antipsychotic treatment on striatal grey matter volume cannot be ruled out. However, independently of the influence of current or previous antipsychotic treatment on striatal grey matter structure, present data suggest a close linkage between striatal functional connectivity and striatal grey matter volume.

\section{Association between connectivity and psychopathology}

In addition, a negative correlation was found between the magnitude of negative symptoms as well as blunted affect and functional connectivity, i.e. the higher the magnitude of negative symptoms and blunted affect, the lower the connectivity between left putamen, anterior cingulate, insula and (ventromedial) frontooccipital areas as well as right putamen, anterior cingulate, insula and middle frontal areas. Here, the significant result in the insula and the anterior cingulate, which together constitute the so-called salience network, may be of major psychopathological relevance given the hypothesis that a disruption within the salience network may constitute the neuronal substrate of blunted affect and passivity in schizophrenia. ${ }^{18}$

Gradin and colleagues' study has recently demonstrated the relevance of functional interplay of midbrain-insular networks with regard to psychopathology. ${ }^{20}$ They found a reduced functional connectivity between midbrain and insula in schizophrenia to correlate with the psychopathological status of the patients. As opposed to our findings, however, Gradin et al detected a significant correlation with severity of psychotic symptoms but no correlation with negative symptoms. More in line with the present findings are recent results by Manoliu et $a l^{49}$ that showed, among others, that a decreased functional connectivity of the left anterior insula correlated with severity of negative symptoms in a sample of people with remitted schizophrenia.

Orliac and colleagues ${ }^{50}$ revealed a decreased functional connectivity in the left and right striatum in a sample of patients with schizophrenia. Somewhat in agreement with our findings they discovered a significant association between connectivity decrease in the left striatum and delusion as well as depression scores. Hence, there is increasing evidence that a disrupted connectivity within reward-related or salience networks is closely linked to characteristic psychopathological symptoms of the disorder.

Whether the impairment in connectivity represents a psychopathological state marker or is to be interpreted in light of an underlying cause of pathology is, however, difficult to determine. Although the significant correlation with the current status or degree of negative symptoms seems to speak in favour of a state marker, the hypothesis that negative symptoms, as a result of an inappropriate allocation of salience to internal representations and external events, constitute the phenomenological consequence of altered neuronal activation in the salience network, would support the latter interpretation. As these questions are difficult to answer given the design of the present study, further, ideally longitudinal studies focusing on the outlined networks should make an attempt to illuminate the significance of altered connectivity within these networks for the psychopathology of schizophrenia.

\section{Limitations}

Given the potential effects of antipsychotic treatment on striatal connectivity and structure the fact that the schizophrenia group 
were taking medication limits the explanatory power of the present study to some degree. In summary, present results suggest a disruption in the functional connectivity of the striatum that is closely linked to striatal volume in people with schizophrenia and that may explain the emergence of negative symptoms.

Kathrin Koch, PhD, Department of Neuroradiology, Klinikum rechts der Isar, Technische Universität München, Munich, Department of Psychiatry and Psychotherapy, Jena University Hospital, Jena, TUM-Neuroimaging Center (TUM-NIC) Psychotherapy, Jena University Hospital, Jena, TUM-Neuroimaging Center (TUM
of Klinikum rechts der Isar, Technische Universität München TUM, Munich and Graduate School of Systemic Neurosciences GSN, Ludwig-Maximilians-Universität, Biocenter, Munich; Oana Georgiana Rus, MA, Tim Jonas Reeß, MA, Department of Neuroradiology, Klinikum rechts der Isar, Technische Universität München, Munich TUM-Neuroimaging Center (TUM-NIC) of Klinikum rechts der Isar, Technische Universität München TUM, Munich and Graduate School of Systemic Neuroscience GSN, Ludwig-Maximilians-Universität, Biocenter, Munich; Claudia Schachtzabel MA, Gerd Wagner, PhD, C. Christoph Schultz, MD, Department of Psychiatry and Psychotherapy, Jena University Hospital, Jena; Christian Sorg, MD, Department of Neuroradiology, Klinikum rechts der Isar, Technische Universität München, Munich; Ralf G. M. Schlösser, Prof. MD, Department of Psychiatry and Psychotherapy, Jena University Hospital, Jena, Germany

Correspondence: Kathrin Koch, Department of Neuroradiology, Klinikum rechts der Isar, Technische Universität München, Ismaningerstrasse 22, 81675 Munich, Germany. Email: kathrin.koch@tum.de

First received 3 Sep 2013, final revision 16 Mar 2014, accepted 28 Mar 2014

\section{Funding}

This work was supported by the German Research Foundation (Deutsche Forschungsgemeinschaft [KO 3744/2-1 to K.K.]).

\section{References}

1 Haber SN, Knutson B. The reward circuit: linking primate anatomy and human imaging. Neuropsychopharmacology 2010; 35: 4-26.

2 O'Doherty J, Dayan P, Schultz J, Deichmann R, Friston K, Dolan RJ. Dissociable roles of ventral and dorsal striatum in instrumental conditioning Science 2004; 304: 452-4.

3 Kahnt T, Park SQ, Cohen MX, Beck A, Heinz A, Wrase J. Dorsal striatalmidbrain connectivity in humans predicts how reinforcements are used to guide decisions. J Cogn Neurosci 2009; 21: 1332-45.

4 Cohen $\mathrm{MX}$, Elger $\mathrm{CE}$, Weber B. Amygdala tractography predicts functional connectivity and learning during feedback-guided decision-making. Neuroimage 2008; 39: 1396-407.

5 O'Doherty JP, Dayan P, Friston K, Critchley H, Dolan RJ. Temporal difference models and reward-related learning in the human brain. Neuron 2003; 38: 329-37

6 Ziauddeen H, Murray GK. The relevance of reward pathways for schizophrenia. Curr Opin Psychiatry 2010; 23: 91-6.

7 Koch K, Schachtzabel C, Wagner G, Schikora J, Schultz C, Reichenbach JR, et al. Altered activation in association with reward-related trial-and-error learning in patients with schizophrenia. Neuroimage 2010; 50: 223-32.

8 Esslinger C, Englisch S, Inta D, Rausch F, Schirmbeck F, Mier D, et al. Ventral striatal activation during attribution of stimulus saliency and reward anticipation is correlated in unmedicated first episode schizophrenia patients. Schizophr Res 2012; 140: 114-21.

9 Morris RW, Vercammen A, Lenroot R, Moore L, Langton JM, Short B, et al. Disambiguating ventral striatum fMRI-related BOLD signal during reward prediction in schizophrenia. Mol Psychiatry 2012; 17: 235, 280-9.

10 Gradin VB, Kumar P, Waiter G, Ahearn T, Stickle C, Milders M, et al. Expected value and prediction error abnormalities in depression and schizophrenia. Brain 2011; 134: 1751-64.

11 Grimm O, Vollstadt-Klein S, Krebs L, Zink M, Smolka MN. Reduced striatal activation during reward anticipation due to appetite-provoking cues in chronic schizophrenia: a fMRI study. Schizophr Res 2012; 134: 151-7.

12 Juckel G, Schlagenhauf F, Koslowski M, Filonov D, Wustenberg T, Villringer A et al. Dysfunction of ventral striatal reward prediction in schizophrenic patients treated with typical, not atypical, neuroleptics. Psychopharmacology 2006; 187: 222-8.

13 Juckel G, Schlagenhauf $F$, Koslowski M, Wustenberg T, Villringer A, Knutson $B$, et al. Dysfunction of ventral striatal reward prediction in schizophrenia. Neuroimage 2006; 29: 409-16.
14 Schlagenhauf F, Juckel G, Koslowski M, Kahnt T, Knutson B, Dembler T, et al. Reward system activation in schizophrenic patients switched from typical neuroleptics to olanzapine. Psychopharmacology 2008; 196: 673-84.

15 Dowd EC, Barch DM. Pavlovian reward prediction and receipt in schizophrenia: relationship to anhedonia. PloS One 2012; 7: e35622.

16 Nielsen MO, Rostrup E, Wulff S, Bak N, Lublin H, Kapur S, et al. Alterations of the brain reward system in antipsychotic naive schizophrenia patients. Biol Psychiatry 2012; 71: 898-905.

17 Walter H, Kammerer H, Frasch K, Spitzer M, Abler B. Altered reward functions in patients on atypical antipsychotic medication in line with the revised dopamine hypothesis of schizophrenia. Psychopharmacology 2009; 206: 121-32.

18 Palaniyappan L, Liddle PF. Does the salience network play a cardinal role in psychosis? An emerging hypothesis of insular dysfunction. J Psychiatry Neurosci 2012; 37: 17-27.

19 Schlagenhauf F, Sterzer P, Schmack K, Ballmaier M, Rapp M, Wrase J, et al. Reward feedback alterations in unmedicated schizophrenia patients: relevance for delusions. Biol Psychiatry 2009; 65: 1032-9.

20 Gradin VB, Waiter G, O'Connor A, Romaniuk L, Stickle C, Matthews K, et al. Salience network-midbrain dysconnectivity and blunted reward signals in schizophrenia. Psychiatry Res 2013; 211: 104-11.

21 Henze R, Brunner R, Thiemann U, Parzer $P$, Richterich $A$, Essig $M$, et al. Gray matter alterations in first-admission adolescents with schizophrenia. J Neuroimaging 2011; 21: 241-6.

22 Mamah D, Wang L, Barch D, de Erausquin GA, Gado M, Csernansky JG. Structural analysis of the basal ganglia in schizophrenia. Schizophr Res 2007; 89: 59-71.

23 Tamagaki C, Sedvall GC, Jonsson EG, Okugawa G, Hall H, Pauli S, et al. Altered white matter/gray matter proportions in the striatum of patients with schizophrenia: a volumetric MRI study. Am J Psychiatry 2005; 162: 2315-21.

24 Kawasaki Y, Suzuki M, Kherif F, Takahashi T, Zhou SY, Nakamura K, et al. Multivariate voxel-based morphometry successfully differentiates schizophrenia patients from healthy controls. Neuroimage 2007; 34: 235-42.

25 Ho BC, Andreasen NC, Ziebell S, Pierson R, Magnotta V. Long-term antipsychotic treatment and brain volumes: a longitudinal study of firstepisode schizophrenia. Arch Gen Psychiatry 2011; 68: 128-37.

26 Molina V, Galindo G, Cortes B, de Herrera AG, Ledo A, Sanz J, et al. Different gray matter patterns in chronic schizophrenia and chronic bipolar disorder patients identified using voxel-based morphometry. Euro Arch Psychiatry Clin Neurosci 2011; 261: 313-22.

27 Ballmaier M, Schlagenhauf F, Toga AW, Gallinat J, Koslowski M, Zoli M, et al. Regional patterns and clinical correlates of basal ganglia morphology in non-medicated schizophrenia. Schizophr Res 2008; 106: 140-7.

28 Keshavan MS, Rosenberg D, Sweeney JA, Pettegrew JW. Decreased caudate volume in neuroleptic-naive psychotic patients. Am J Psychiatry 1998; 155: 774-8.

29 American Psychiatric Association. Diagnostic and Statistical Manual of Mental Disorder (4th edn) (DSM-IV). APA, 1994.

30 Annett $M$. The binomial distribution of right, mixed and left handedness. Q J Exp Psychol 1967; 19: 327-33.

31 First MB, Spitzer RL, Gibbon M, Williams JBW. Structured Clinical Interview for DSM-IV Axis I Disorders, Clinician Version (SCID-CV). American Psychiatric Press, 1996.

32 Woods SW. Chlorpromazine equivalent doses for the newer atypical antipsychotics. J Clin Psychiatry 2003; 64: 663-7.

33 Kay SR, Fiszbein A, Opler LA. The positive and negative syndrome scale (PANSS) for schizophrenia. Schizophr Bull 1987; 13: 261-76.

34 Sutton RS, Barto AG. Reinforcement Learning: An Introduction. MIT Press, 1998.

35 Dayan $\mathrm{P}$, Abbott LF. Theoretical Neuroscience Computational and Mathematical Modeling of Neural Systems. MIT Press, 2001.

36 Niv Y, Schoenbaum G. Dialogues on prediction errors. Trends Cogn Sci 2008; 12: 265-72.

37 Friston KJ, Buechel C, Fink GR, Morris J, Rolls E, Dolan RJ. Psychophysiological and modulatory interactions in neuroimaging. Neuroimage 1997; 6: 218-29.

38 Rijcken CA, Monster TB, Brouwers JR, de Jong-van den Berg LT. Chlorpromazine equivalents versus defined daily doses: how to compare antipsychotic drug doses? J Clin Psychopharmacology 2003; 23: 657-9.

39 Yilmaz A, Simsek F, Gonul AS. Reduced reward-related probability learning in schizophrenia patients. Neuropsychiatr Dis Treat 2012; 8: 27-34.

40 Murray GK, Corlett PR, Clark L, Pessiglione M, Blackwell AD, Honey G, et al. Substantia nigra/ventral tegmental reward prediction error disruption in psychosis. Mol Psychiatry 2008; 13: 239, 267-76. 
41 Seger CA. How do the basal ganglia contribute to categorization? Their roles in generalization, response selection, and learning via feedback. Neurosci Biobehav Rev 2008; 32: 265-78.

42 Cooper JC, Knutson B. Valence and salience contribute to nucleus accumbens activation. Neuroimage 2008; 39: 538-47.

43 Chikama M, McFarland NR, Amaral DG, Haber SN. Insular cortical projections to functional regions of the striatum correlate with cortical cytoarchitectonic organization in the primate. J Neurosci 1997; 17: 9686-705.

44 Cauda F, D'Agata F, Sacco K, Duca S, Geminiani G, Vercelli A. Functional connectivity of the insula in the resting brain. Neuroimage 2011; 55: 8-23.

45 Wylie KP, Tregellas JR. The role of the insula in schizophrenia. Schizophr Res 2010; 123: 93-104

46 Duerden EG, Arsalidou M, Lee M, Taylor MJ. Lateralization of affective processing in the insula. Neuroimage 2013; 78: 159-75.
47 Leichnetz GR. Connections of the medial posterior parietal cortex (area $7 \mathrm{~m}$ ) in the monkey. Anat Rec 2001; 263: 215-36.

48 Bora E, Fornito A, Radua J, Walterfang M, Seal M, Wood SJ, et al. Neuroanatomical abnormalities in schizophrenia: a multimodal voxelwise meta-analysis and meta-regression analysis. Schizophr Res 2011; 127: 46-57.

49 Manoliu A, Riedl V, Doll A, Bauml JG, Muhlau M, Schwerthoffer D, et al. Insular dysfunction reflects altered between-network connectivity and severity of negative symptoms in schizophrenia during psychotic remission. Front Hum Neurosci 2013; 7: 216

50 Orliac F, Naveau M, Joliot M, Delcroix N, Razafimandimby A, Brazo P, et al. Links among resting-state default-mode network, salience network, and symptomatology in schizophrenia. Schizophr Res 2013; 148: 74-80.

\section{On Bereavement: Studies of Grief in Adult Life by Colin Murray Parkes}

\section{Jan Oyebode}

The first edition of Colin Murray Parkes' Bereavement: Studies of Grief in Adult Life was published in 1972, coinciding with the first year of my studies towards a degree in psychology at the University of Liverpool. These were the high days in psychology of 'positivist empiricism'. We were concerned for our subject to be taken seriously as a science, and aimed to do this through the development of an experimental evidence base for the application of psychology, lest we be dismissed as mere armchair philosophers. Secretly, however, many of us students were somewhat disappointed to find that our subject disaggregated people into small parts. We spent our time on topics such as list learning of nonsense syllables or the pecking behaviour of pigeons, but harboured a wish to know about people and what makes them tick. Colin Murray Parkes' book, with its holistic narrative descriptions of bereaved widows, fed this desire to think about people as whole - feeling, thinking, reacting - beings. The descriptions of bereaved women, in their social, cultural and family contexts, brought the phenomena of bereavement alive in a way that a dry textbook could not have done. This concentration on detailed individual descriptions presaged the phenomenal increase of interest in phenomenology and understanding of subjective experience that followed over the next 20 years but, at the time, it was rare.

The power of personal vignettes was demonstrated by my experience some years later when, as a recently qualified clinical psychologist, I turned to the book to help inform a workshop I had been invited to deliver to care staff who worked with older people. Being young and naîve, I had not appreciated the impact Colin's rich descriptions might have on a predominantly middle-aged audience of women, many of whom dissolved into tears as I spoke. Since those days, I have hopefully matured in my approach to teaching but have continued to draw on material in the book. Similarly, later editions of the book have reflected the maturing range of research and theory about bereavement. They retain the descriptions at their heart but the wider context has been updated to ensure the book retains contemporary relevance.

Sadly, my first edition walked from my shelves many years ago, no doubt lent by me to an enthusiastic trainee clinical psychologist who now has it on their own shelf, unless it has been, in turn, passed on to another. Re-reading my third edition, in addition to the rich descriptions, I was struck by the scholarly integration of material spanning arts and science, drawn from historical and literary sources, as well as psychiatry, psychology, sociology and ethology. I also noticed the author's voice coming through in the text, with a gift for demystifying the complex, making ideas accessible. He discloses valuable lessons from personal experience, expressed in an unassuming way, which offer wise advice to those of us who work with those who have experienced loss. 